\title{
Dual effects of 'losses disguised as wins' and near- misses in a slot machine game
}

\section{Steve Sharman, Michael RF Aitken \& Luke Clark}

To cite this article: Steve Sharman, Michael RF Aitken \& Luke Clark (2015) Dual effects of 'losses disguised as wins' and near-misses in a slot machine game, International Gambling Studies, 15:2, 212-223, DOI: 10.1080/14459795.2015.1020959

To link to this article: http://dx.doi.org/10.1080/14459795.2015.1020959

\section{曲 Published online: 27 Mar 2015.}

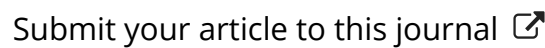

Џ Article views: 147

Q View related articles $₫$

View Crossmark data $\nearrow$ 


\title{
Dual effects of 'losses disguised as wins' and near-misses in a slot machine game
}

\author{
Steve Sharman ${ }^{\mathrm{a} *}$, Michael RF Aitken ${ }^{\mathrm{a}, \mathrm{b}}$ and Luke Clark $^{\mathrm{c}}$ \\ ${ }^{a}$ Department of Psychology, University of Cambridge, Cambridge, UK; ${ }^{b}$ Institute of Psychiatry, \\ Kings College London, London, UK, ${ }^{c}$ Centre for Gambling Research at UBC, Department of \\ Psychology, University of British Columbia, Vancouver, Canada
}

(Received 1 September 2014; accepted 15 February 2015)

\begin{abstract}
Individually, both near-misses and losses disguised as wins (LDWs) have been seen to exert pro-motivational effects on gambling. However, it is not clear whether both structural characteristics are effective within the same game. Participants $(n=40)$ played a slot machine simulation. The simulation delivered near-misses, wins and 'fullmisses'. Half the participants also received LDWs that occurred independently of the outcomes on the payline. Valence and motivation ratings were collected after each round. Results showed that the LDW group reported increased valence ratings compared to the no-LDW group. Within the LDW group, trials with LDWs also resulted in increased enjoyment compared to trials without LDWs. We distinguished near-misses falling either side of the payline. Near-misses before the payline (NMB) were rated as more motivational than near-misses after the payline (NMA), whereas NMAs were rated as more aversive than NMBs. These differences between the two near-miss types were exacerbated by LDWs. Results demonstrate LDWs increase the trial-by-trial enjoyment of non-win outcomes. The motivational and hedonic effects of near-misses differed for events either side of the payline, and these differences were exaggerated by the presence of LDWs. Thus, near-misses can retain their effectiveness in complex forms of gambling that also deliver LDWs.
\end{abstract}

Keywords: addiction; gambling; problem gambling; electronic machine gambling; near-miss; cognition

\section{Introduction}

Structural characteristics of contemporary electronic gaming machines (EGMs) correspond to psychological processes that are linked to players' enjoyment of the game, persistent play and risk of developing gambling problems (Griffiths, 1993; Meyer, Fiebig, Häfeli, \& Mörsen, 2011). Modern commercial games are increasingly complex, combining many different structural characteristics in the same game (Dow Schüll, 2012). Laboratory studies with simplified EGM 'simulations' provide an important means of delineating the behavioural effects of individual structural characteristics in a controlled environment, such as immediacy of reward (Chóliz, 2010), the role of early wins and unreinforced trials (Haw, 2008), and the relationship between big wins and gambling persistence (Weatherly, Sauter, \& King, 2004).

One widely discussed structural characteristic is the near-miss: a non-win outcome where the wager is lost but the outcome is perceived as proximal to a major payout (Parke $\&$ Griffiths, 2004). Near-misses are pervasive across all forms of gambling, and are usually signified by spatial proximity; for example, two cherries on the payline with the third

*Corresponding author. Email: sps48@cam.ac.uk 
cherry falling adjacent to the payline. A moderate rate of near-misses is known to increase gambling persistence: a group of video lottery players who played a game with a $27 \%$ rate of near-misses played for 33\% longer than a group with no near-misses (Côté, Caron, Aubert, Desrochers, \& Ladouceur, 2003). This persistence peaks when near-misses are experienced on $30 \%$ of trials, compared to lower $(15 \%)$ or higher $(45 \%)$ rates (Kassinove $\&$ Schare, 2001). On a commercial slot machine, participants report that near-misses felt closer to wins than other non-win outcomes (which we term 'full-misses'; Dixon \& Schreiber, 2004). Using psychophysiological monitoring, near-misses are seen to be more arousing than full-misses in terms of skin conductance levels (Clark, Crooks, Clarke, Aitken, \& Dunn, 2012; Dixon et al., 2011), despite their status as objectively-equivalent outcomes.

One of the major difficulties with studying near-misses in naturalistic settings is the number of different near-miss types that can occur. Using a classic three-reel machine with a sequential stop, the payline alone offers three possible combinations (XXO, XOX, $\mathrm{OXX}$ ). An early paper saw greater persistence in a group receiving the XXO type than the OXX type (Strickland \& Grote, 1967), and this finding was extended to psychophysiological arousal measures by Dixon et al. (2013a). XXO reel configurations also exerted differential motivational effects (to XOX configurations) on decisions to take a second chance to 'repair the loss' (Bossuyt, Moors, \& De Houwer, 2014). Most slot machines offer a larger 'field of view' that displays lines adjacent to the payline as well, enabling near-misses where the matching either stops just short, or passes through the payline to the next position. It is likely that these kinds of near events may also differ in their effects. In previous work using a simplified two-reel slot machine, near-misses were rated as more unpleasant than full-misses, but simultaneously increased participant's desire to continue playing more than full-misses (Billieux, Van der Linden, Khazaal, Zullino, \& Clark, 2012; Clark, Lawrence, Astley-Jones, \& Gray, 2009; Qi, Ding, Song, \& Yang, 2011). Separating near-misses either side of the payline, the motivational effect was strongest on near-misses before the payline (NMBs), and the aversive effect was strongest on the near-misses after the payline (NMAs) (Clark et al., 2012). These differences may be mediated by differential behavioural effects of 'additive' counterfactual thoughts (which are behaviourally activating) versus 'subtractive' counterfactuals (i.e. mentally 'un-doing' reality), which generates an 'expectancy violation' (Roese \& Olson, 1995a) and 'affective contrast' (Markman \& McMullen, 2003).

LDWs represent a distinct phenomenon to near-misses, and are a further characteristic of modern multi-line slot machines. Critically, these games offer players the opportunity to simultaneously bet on multiple 'win lines' on any given spin. A win on one (or more) lines will trigger a payout, plus the sensory feedback of winning, but the amount won is often less than the total stake, resulting in a net loss. When playing these games, regular EGM gamblers often adopt the mini-max strategy, placing the minimum bet on the maximum number of lines, which serves to increase the cost of each spin, and the number of win opportunities. This strategy maximizes the number of LDWs without affecting the actual return to player (Harrigan, Dixon, MacLaren, Collins, \& Fugelsang, 2011). In a sample of regular gamblers (including problem gamblers), $94 \%$ preferred a multi-line simulator over a structurally equivalent single-line game (Dixon et al., 2014). Moreover, while these effects have been discussed almost exclusively within the context of multi-line slot machines, the same phenomenon is present in some other games, including electronic roulette, which is reported as the most common form of gambling among British treatment-seeking pathological gamblers (Michalczuk, Bowden-Jones, Verdejo-Garcia, \& Clark, 2011). In roulette, a gambler can place multiple wagers on various combinations 
(odd/even, red/black, individual numbers etc.), but a single win may not recoup the initial outlay, generating an LDW.

Physiologically, LDWs have been shown to evoke raised skin conductance responses compared to full-misses. The LDW response is similar in magnitude to the response to actual wins (Dixon, Harrigan, Sandhu, Collins, \& Fugelsang, 2010) and also generates a 'post reinforcement pause', which is seen as a consummatory response to reward (Dixon et al., 2014). In a notable study, Jensen et al. (2013) found that LDWs were verbally miscategorized as wins, and that players' estimates of the number of wins after the game were higher when they were exposed to more LDWs. This mis-categorization of LDWs as normal wins (presumably because of the equivalent sensory feedback) serves to reduce the length of unbroken loss streaks and increase the frequency of reinforcement (every 2.1 spins, versus 6.5 spins in a single-line game), whilst the average proportion of stake that is returned to the player through wins (the payback percentage) remains the same (Harrigan et al., 2011). LDWs may foster the dissociative state termed the 'machine zone' (Dow Schull 2012) and pathological gamblers may be disproportionately sensitive to this immersive effect (Dixon et al., 2014).

A key difference between near-misses and LDWs is that LDWs generate the typical sensory feedback (the 'bells and whistles') of winning, whereas near-misses do not. Removal of sounds from a slot machine game has been shown to decrease enjoyment, and the excitement of slot machine play (Dixon et al., 2015; Loba, Stewart, Klein, \& Blackburn, 2001). While LDWs increase post-reinforcement pauses (Dixon et al., 2014), near-misses do not (Dixon et al., 2013b).

Despite the growing body of work looking at near-misses and LDWs, the two effects have not been examined within the same task. The current study sought to compare the effects of near-misses delivered in the presence or absence of LDWs. Given the frequent reinforcement and intense sensory feedback associated with LDWs, one possibility is that near-misses may be rendered less effective in the modern games. Thus, we hypothesized that the difference in subjective ratings between near-miss and full-miss outcomes would be attenuated in a group who also experienced LDWs. However, we also recognized $a$ priori that an alternative pattern of results was possible, whereby the effects of LDWs may serve to invigorate overall behavior on the task and thus heighten the effects of other game events, including near-misses. The current study used a three-reel simulation to deliver LDWs independently from the payline outcomes (wins, near-misses and full-misses). We compared a group of participants who experienced LDWs against a second group who did not, and compared responses to the near-miss types between groups. As a second set of analyses in the group receiving LDWs, we compared the different outcome types in the presence or absence of a LDW.

\section{Method}

\section{Participants}

Forty participants were randomly assigned to an LDW group ( $n=20$ (13 male), age $\mathrm{M}=23, \mathrm{SD}=8.9)$, or a no-LDW group $(n=20(10$ male $)$, age $\mathrm{M}=20.5, \mathrm{SD}=$ 1.05). Participants were recruited through university mailing lists and within-college advertising, and were mainly students with limited gambling experience. The groups did not differ on age $(t(38)=1.24, p=.22)$ or gender distribution $\left(\chi^{2}(1)=.921, p=\right.$ .34). For screening purposes, participants completed the Problem Gambling Severity Index (PGSI; Ferris \& Wynne, 2001), and the majority of participants scored zero (LDW group: $10,(\mathrm{M}=1.2, \mathrm{SD}=1.7)$; no-LDW group: $13(\mathrm{M}=0.4, \mathrm{SD}=0.6)$ ). 
To examine group differences in gambling-related traits, participants completed the Gambling Related Cognitions Scale (GRCS; Raylu \& Oei, 2004) and the UPPS-P Impulsive Behavior Scale (UPPS; Cyders et al., 2007); the groups did not differ significantly on either measure: GRCS, (LDW group $\mathrm{M}=54.9, \mathrm{SD}=19.8$; no-LDW: $\mathrm{M}=46.3, \mathrm{SD}=16.5 ; t(38)=1.49, \mathrm{p}=.144)$, UPPS-P scores (LDW M $=124.5$, $\mathrm{SD}=24$; no-LDW: $\mathrm{M}=133.9, \mathrm{SD}=19.1 ; t(38)=1.38, \mathrm{p}=.174)$. Ethical approval for the experiment was obtained from the Cambridge Psychology Research Ethics Committee. Participants provided written consent, before completing the questionnaires. Participants were read tailored instructions for each group, before playing the slot machine game on a Dell desktop computer. Participants were paid $£ 4$ to take part in the research, plus the remaining balance on the slot simulator at the end of the task.

\section{Task design}

The task involved a three-reel slot machine simulation. The background theme of the simulation was the Wild West. The icons on the reels included items such as a pistol, a rusty sheriff's badge and a cowboy hat. Sound effects on the task were also Wild West themed and were consistent across groups. The win line was the horizontal centre line of a $3 \times 3$ matrix, allowing 9 icons to be in view. A win occurred when three matching icons were stopped on the win line. Each icon was surrounded by a square coloured border, (either red, green, blue, black, orange or yellow). Rather than having multi-line bets, LDWs occurred when 3 or more of the 9 coloured borders matched. This involved similar attentional processing to the diagonal and zig-zag combinations as in multi-line games. For the no-LDW group, the border colours were still present to equate sensory load, but had no financial significance.

All participants started the task with an endowment of 5000 credits, equivalent to $£ 5$. In the LDW group, a spin cost 150 credits, an LDW was worth 100 credits, and a win was worth 3000 credits (or 3100 credits when it coincided with three border colours matching). In the no-LDW group, each spin cost 100 credits, and a win was worth 3000 credits. This payout structure, combined with a fixed set of outcomes, ensured that each participant finished the task with 4000 credits (a bonus of $£ 4$ ), and maintained a consistent payback percentage across groups. The task satisfies criteria for gambling through requiring the participants to wager credits (with real monetary value) in the hope of obtaining a greater monetary win. Participants were informed that their final balance on the task would be honoured as a bonus payment, and thus were personally invested in the task.

The task used a nested trial structure, so that each block of trials began with a 'full spin' where all three reels spun. Following the full spin (which never produced a win) the participant chose one reel to hold. The participant then had up to five double spins (of the two non-held reels) to match a second reel, which was then held automatically. A 'final spin' occurred after two matching icons had been held, which was accompanied by a western-themed tune and the words 'last chance saloon' (Figure 1). This final spin led to one of three outcomes (win, near-miss or full-miss). After the outcome of the final spin, or after an unsuccessful series of double spins, all reels were 'unheld', and the next spin was a full spin. Our analysis therefore focused on the 24 'final spins'.

The introduction of the hold feature into the task served a dual purpose; first, it allowed the participant to choose which icon to hold; past work has shown that near-misses are more effective when personal control is present (Clark et al., 2009). Second, the hold function served to limit the number of different near-miss types to those where a single reel stops adjacent to two matching reels. 


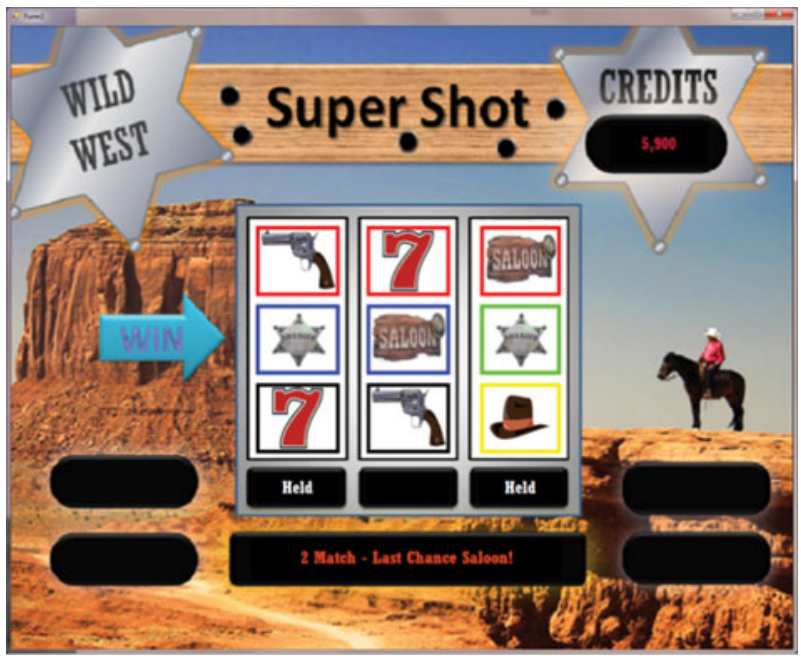

Figure 1. Task screen shot.

Participants played 124 spins in total, comprising 24 final spins, which thus occurred approximately every 5 spins. A full-miss on a final spin occurred when the icon matching the two held reels did not finish in view on the third reel. Near-misses occurred when the third reel stopped with the desired icon (matching the two held reels) one square before the payline (NMB, Figure 2a), or one square after the payline (NMA, Figure 2b). The stimuli shown on the first spin on each trial were counterbalanced across participants, with the stimuli presented on second and subsequent spins on a trial determined by the selection of the participant on the initial spin in order to produce the final outcome of the trial (win, full miss, NMA, NMB). The order of final outcomes across was randomized for each participant. For the LDW group, LDWs occurred on 50\% of trials and could occur on any spin, not just the final spin. LDWs were signified by the words 'three color match', and the same western-themed win jingle as a win.

\section{Ratings}

After each of the final spins, participants were presented with two ratings. To measure outcome valence, participants were asked 'How happy are you with that result'? Participants answered on a 100 point sliding scale, ranging from 'Not at all happy' (0) to 'Very happy indeed' (100). To measure motivation to continue playing, participants were asked 'How much do you want to continue playing?'; responses were given on a 100 point

(a)

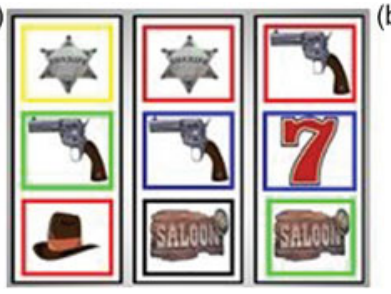

(b)

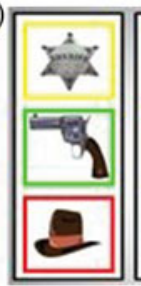

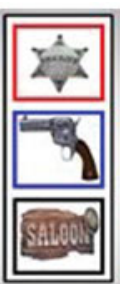
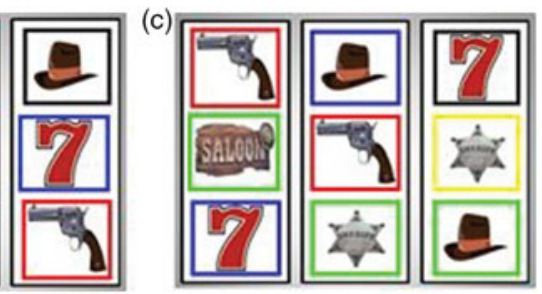

Figure 2. Outcomes (a) Near-miss before (b) Near-miss after (c) Full-miss. 
sliding scale, ranging from 'Not at all' (0) to 'Very much' (100). By acquiring ratings every five spins (approximately) as opposed to every trial (Clark et al., 2009), participants experienced longer blocks of continuous play, which we considered to be more reflective of real slot machine play.

\section{Statistical analysis}

Scores on the ratings scales were coded in the output files as proportion values from 0 to 1 (i.e. a rating of 49 became a score of .49). For the valence and motivation ratings, an omnibus mixed model ANOVA was run with a repeated-measure factor of Outcome (win, NMA, NMB, full-miss) and a between-subjects factor of Group (LDW, no-LDW). A secondary model looked at ratings in a model restricted to the three non-win outcome types. A further model looked at ratings within the LDW group on trials with and without LDWs. All data were checked for homogeneity of variance and Greenhouse-Geisser corrected where appropriate. Alpha was set at 0.05 for all tests. Error bars represent the standard error of the mean.

\section{Results}

\section{Omnibus models}

The omnibus ANOVA model for valence ratings indicated a significant main effect of Outcome $\left(\mathrm{F}(1.4,53.3)=182.2, \mathrm{p}<.001, \eta^{2}=.83\right)$. Collapsed across groups, valence ratings were higher following wins than the three non-win outcome types (smallest $t$ value $=14.06)$. The tendency for higher ratings in the LDW group did not reach significance (main effect of Group: $\left.\mathrm{F}(1,38)=3.6, \mathrm{p}=.065, \eta^{2}=.087\right)$, nor was there a tendency for this effect to vary with Outcome type (Outcome x Group: $\mathrm{F}(1.4,53.3)=1.2, \mathrm{p}=$ $\left..29, \eta^{2}=.031\right)$. The equivalent model for the motivation rating indicated a significant main effect of Outcome $\left(\mathrm{F}(1.7,63.9)=69.5, \mathrm{p}<.001, \eta^{2}=.65\right)$. Collapsed across groups, the effect was driven by higher ratings following wins than the other three non-win outcome types (smallest $t$ value $=7.27$ ). There was no evidence of a difference between the groups, nor any Outcome x Group interaction (larger $\mathrm{F}(1,38)=$ $\left.1.7, \mathrm{p}=.19, \eta^{2}=.04\right)$.

Given that the winning outcomes differ from other outcomes in their objective reward, a restricted model was run to compare the three non-win outcomes directly. For valence ratings, the Outcome $x$ Group interaction was significant $\left(F(2,76)=4.01, p=.02, \eta^{2}\right.$ $=.096)$, as was the main effect of Outcome $(\mathrm{F}(2,76)=15.8, \mathrm{p}<.001)$ with lower valence ratings across groups following NMAs than other non-win outcomes (smaller $t$ value $=3.87)$. The main effect of Group was not significant $(\mathrm{F}(1,38)=2.7, \mathrm{p}=.11$, $\left.\eta^{2}=.07\right)$. Further investigation of the interaction confirmed that the difference in valence ratings between the two types of near-misses was greater in the LDW group (M $=.10, \mathrm{SD} .12)$ than the no-LDW group $(\mathrm{M}=.02, \mathrm{SD} .05)(t(38)=2.56, \mathrm{p}=.014)$. The LDW group reported increased valence ratings following NMBs compared to the noLDW group $(t(38)=2.57, \mathrm{p}=.014)$. The groups did not differ significantly on valence ratings following NMAs $(t(38)=.69 \mathrm{p}=.49)$ or full-misses $(t(38)=1.7, \mathrm{p}=.11)$, see Figure 3a.

The equivalent model for the motivation ratings yielded a significant main effect of Outcome $(\mathrm{F}(2,76)=40.1, \mathrm{p}<.001)$; there was no significant main effect of Group ( $\mathrm{F}$ $(1,38)=2.04, \mathrm{p}=.162)$ or Outcome $\mathrm{x}$ Group interaction $(\mathrm{F}(2,76)=.16, \mathrm{p}=.86)$. Collapsed across group, NMBs $(\mathrm{M}=.49, \mathrm{SD}=.18)$ generated an increased motivation 

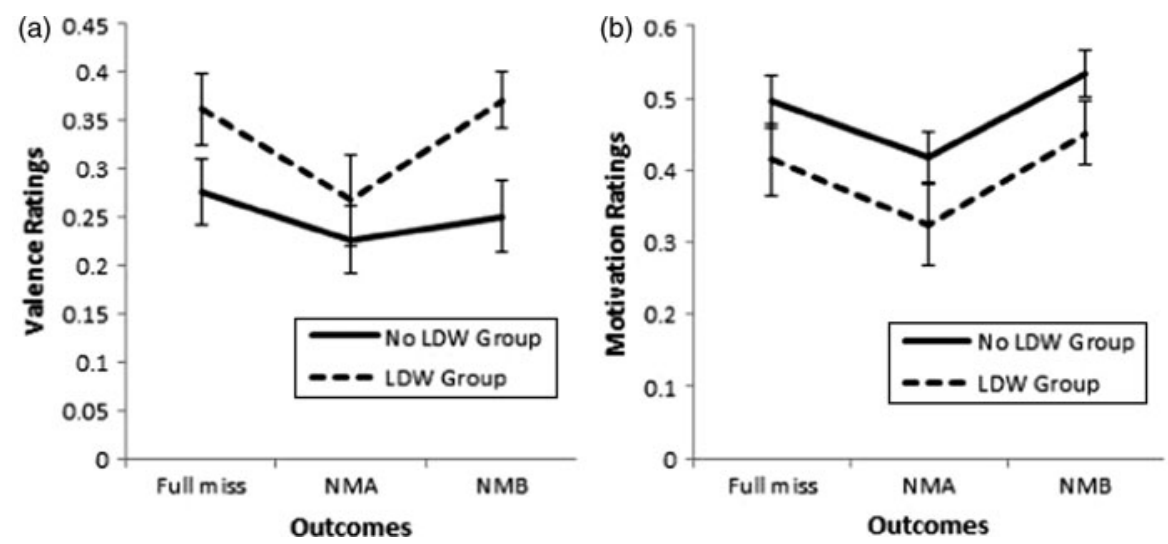

Figure 3. Valence and motivation ratings between groups.

to continue compared to NMAs $(\mathrm{M}=.37, \mathrm{SD}=.21 ; t(39)=7.9, \mathrm{p}<.001)$ and full-misses $(\mathrm{M}=.45, \mathrm{SD}=.20 ; t(39)=3.3, \mathrm{p}=.002)$. Full-misses were rated as more motivational than NMAs $(t(39)=5.9, \mathrm{p}<.001)$, see Figure $3 \mathrm{~b}$.

\section{Within the LDW group}

The next set of analyses focused within the LDW group comparing trials where LDWs were delivered against trials without LDWs. On the valence ratings, an ANOVA model with Outcome and LDW (present, absent) as factors confirmed that outcomes that were accompanied by a LDW were rated as significantly more pleasing than trials without a LDW (main effect of LDW: $\mathrm{F}(1,19)=17.8, \mathrm{p}<.001$ ), and different outcome types received different ratings $(\mathrm{F}(2,38)=10.4, \mathrm{p}<.001)$. The difference in valence rating varied across outcomes (LDW x Outcome interaction: $\mathrm{F}(2,38)=12.3, \mathrm{p}<.001)$; the difference in valence ratings between the two near-miss types was greater on LDW trials $(\mathrm{M}=.17, \mathrm{SD} .16)$ than on trials without LDWs $(\mathrm{M}=.04, \mathrm{SD} .12),(t(19)=5.04$, $\mathrm{p}<.001$ ), see Figure 4a.

The equivalent model for the motivation ratings showed that ratings varied across the different types of outcome (main effect of Outcome: $\mathrm{F}(2,38)=20.7, \mathrm{p}<.001$ ). The
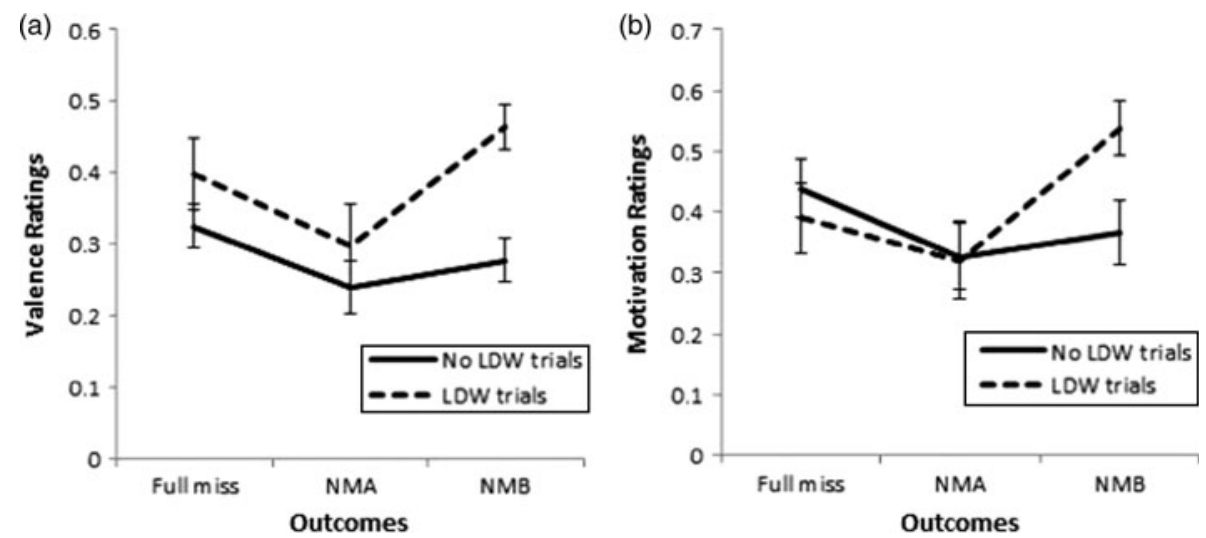

Figure 4. Valence and motivation ratings within the LDW group. 
presence of LDWs had no reliable overall effect (main effect of LDW: $F(1,19)=2.2$, $\mathrm{p}=.156$ ) but LDWs had a differential effect across the three outcomes (LDW $\mathrm{x}$ Outcome: $\mathrm{F}(2,38)=12.3, \mathrm{p}<.001)$. The difference in motivation ratings following the two near-miss types (NMB producing higher motivation ratings than NMA) was larger when these outcomes were accompanied by LDWs $(\mathrm{M}=.22$, SD $=.16)$ than in nearmiss trials without LDWs $(\mathrm{M}=.04, \mathrm{SD} .10)(t(19)=4.6, \mathrm{p}<.001)$, see Figure $4 \mathrm{~b}$.

\section{Discussion}

Using a three-reel slot machine simulation, we sought to explore the interplay between the effects arising from two structural characteristics of a gambling game, the near-miss and the LDW. Specifically, we investigated whether the presence of LDWs increased or decreased the subjective effects of near-misses when both events occurred in the same task. We saw that wins exerted a significant and strong effect on the ratings of both 'pleased with outcome' and 'continue to play', in a way that was similar in the groups who did and did not receive LDWs. We also observed that the subjective effects of near-misses depended on their position relative to the payline, consistent with previous work using a less realistic two-reel slot machine simulation (Clark et al., 2009, 2012). Across both groups, relative to other non-win outcomes, near-misses that stopped before the payline (NMB) increased the motivation to continue the game, whereas near-misses that passed through the payline (NMA) were rated as less pleasing (i.e. more aversive).

The group that experienced LDWs afforded higher valence ratings to all non-win outcomes compared to the group that did not experience LDWs. Within the group that experienced LDWs, LDW trials were also rated as more enjoyable than trials without LDWs. Thus, despite being net losses, and making no difference to the overall rate of payout, the occurrence of LDWs acted to increase the trial-by-trial enjoyment of the game. Previous studies have indicated that LDWs induce elevated skin conductance responses compared to other non-win outcomes, related to the sensory feedback associated with winning (Dixon et al., 2010). Our results extend this work to show elevated valence responses to trials with LDWs, such that the presence of LDWs increases the enjoyment of non-win outcomes. The noises and jingles that co-occur with wins are known to influence players' preferences for different machines, influence both psychological and physiological arousal, and cause players to overestimate how many times they won (Dixon et al., 2013a). As observed by Harrigan et al. (2011), increasing the number of win opportunities increases the frequency with which positive reinforcement of gameplay via auditory and visual feedback associated with wins can be delivered. Thus, our results indicate that the increased frequency of reinforcement cues afforded by LDWs directly increases the overall enjoyment associated with gameplay, despite maintaining a consistent payback percentage and resulting in an objective overall loss.

The presence of LDWs exaggerated the differences between the two near-miss types. Within-subject increases in motivation to continue were larger following NMBs relative to NMAs on LDW trials, compared to trials without LDWs. A similar difference was observed in between-groups analysis, where the motivational effect was larger for the group experiencing LDWs than for the group who did not. Additionally, NMAs were rated as significantly more aversive than NMBs in the presence of LDWs, both within and between groups, with the larger effect seen within the LDW group. Thus, contrary to our primary hypothesis, LDWs enhanced both the motivational effect of the NMBs and the aversive effect of the NMAs indicating the presence of LDWs invigorated overall behavior and increased attention to, and the salience of, all other game events. 
The increased motivation to continue playing following NMBs may be a result of counterfactual thinking; when a player experiences a NMB, the nearly-obtained win is an additive counterfactual, in that the obtained outcome is compared with a win that was yet to come (Roese, 1997). This allows the player to believe the NMBs are indicative that a win or reward is due, thus increasing the motivation to continue playing. The motivation is increased in the presence of LDWs. This may arise from association of the sensory feedback generated by LDWs with the actual wins, or because the 'win' (of an amount lower than the stake) reduces the negative aspect of perceiving the near-miss as an objective loss.

NMAs were rated as significantly more aversive than NMBs, a difference that was exaggerated in the presence of LDWs. When a player experiences a NMA, the reels are presented in a winning configuration immediately prior to the outcome; this may create a larger anticipatory expectation of winning than at any point on a NMB trial (where the win configuration is never presented). The final movement of the reel which produces the NMA configuration means that the anticipated win does not occur, producing an 'expectancy violation' (Roese \& Olson, 1995a). The expectancy violation produces negative affect, as the expected reward is denied, leading to NMAs being rated as more aversive than NMB outcomes. The enhancement of this effect in the presence of LDWs may be explained by consideration of the small 'win' within the LDW. On NMA trials, the LDW outcome 'small win' is smaller than the anticipated gain, and thus may be regarded as a negative expectancy violation. By contrast, on NMB trials (where the large anticipatory expectation does not occur), the LDW outcome is a positive expectancy violation (as the outcome is better than a pure loss).

Recent work modelling near-miss effects in the laboratory has established a number of important findings: (i) these outcomes generate physiological arousal, brain reward responses, and motivate continued play (Clark et al., 2009; Dixon et al., 2011); (ii) problem gamblers, and individuals with higher trait susceptibility to gambling distortions, are more reactive to near-misses (Billieux et al., 2012; Chase \& Clark, 2010; Dymond et al., 2014). However, it is also noted that modern games are so complex that the opportunities for near-misses are almost boundless, and other features like LDWs may be increasingly important. Our results show that increasing game complexity (at least in terms of reinforcement frequency and sensory feedback) does not diminish the traditional near-miss effect; rather it enhances it. As such, near-miss features may still warrant consideration in risk assessment tools (Meyer et al., 2011) and future gambling legislation.

\section{Limitations}

Laboratory simulations of gambling games allow controlled isolation of structural characteristics, but also involve notable compromises. As strengths, we can specify outcome sequences to deliver equal numbers of key events (wins, LDWs, near-misses) across all participants, and specify their distribution over the duration of the task (cf. Langer \& Roth, 1975). By playing the task in a lab without other gambling machines in proximity, we avoid social influences such as the sight and sound of players winning on adjacent machines (the 'co-action' effect; Rockloff \& Greer, 2011). Nevertheless, the ecological validity of the task is reduced by removing some elements of choice that would be available on a commercial machine; for example, participants were not able to vary the number of lines or vary their stake. (We note that in Dixon et al.'s (2010) experiment with a multi-line simulator, they also forced participants to place the maximum bet on all available lines on each trial.) Additionally, the measures of valence and motivation were 
provided by the participants on a self-report scale; although the task was structured to administer these rating on average every fifth spin, it is likely that this is nonetheless sufficient to interrupt the 'machine zone' (Dow Schüll, 2012). Extrapolating these results to continuous play should therefore only be done with caution. Participants in our study were gambling novices, and further work is needed to establish whether these effects generalize to other levels of gambling involvement, including pathological gambling. The availability of monetary reward is an important prerequisite for ecological validity in gambling research (e.g. Anderson \& Brown, 1984; Ladouceur, Sévigny, Blaszczynski, O'Connor, \& Lavoie, 2003). As a laboratory study, we adopted principles from behavioural economics that participants were endowed a sum of the money to play the game, and the remaining balance upon completion of the task was honored in the form of a bonus payment. Thus the task satisfies criteria for gambling through requiring the participants to wager some of their endowed funds in the hope of obtaining a larger monetary win. Nevertheless, endowed funds may be considered 'house money' (e.g. Thaler \& Johnson, 1990) and participants could perform differently under conditions where they were voluntarily risking their own funds.

In conclusion, this study provides laboratory evidence that the presence of LDWs within a slot machine task increases the positive valence following non-win outcomes. Second, the presence of LDWs exaggerates the difference in both valence and motivation ratings between the two types of near-misses. In tandem, these constructs can increase the motivation to continue playing, and the enjoyment of a particular form of gambling when the individual is losing.

\section{Conflicts of interest}

Funding sources: SS was funded by a graduate scholarship from the University of Cambridge. Funding sources had no involvement in the study design, collection, analysis or interpretation of data, or in the writing of the report.

Competing interests: The Centre for Gambling Research at UBC is supported by an award from the British Columbia Lottery Corporation and the British Columbia Government. LC acknowledges funding from the Medical Research Council (G1100554). MRFA has no competing interest to declare.

Constraints on publishing: There are no constraints on publishing.

\section{Notes on contributors}

Steve Sharman is a PhD student within the Department of Psychology at the University of Cambridge. His interests include the role of within game constructs on problem and non-problem gambling behavior, and problem gambling in vulnerable populations.

Mike Aitken is a reader in psychology at the Institute of Psychiatry, Psychology and Neuroscience at King's College London. His interests include judgments and decisions concerning uncertainty, including gambling behavior.

Luke Clark is the director of the Centre for Gambling Research at UBC. His research interests include the psychological and neural basis of cognitive distortions in gambling and gambling addiction.

\section{References}

Anderson, G., \& Brown, R. I. F. (1984). Real and laboratory gambling, sensation-seeking and arousal. British Journal of Psychology, 75, 401-410. doi:10.1111/j.2044-8295.1984.tb01910.x 
Billieux, J., Van der Linden, M., Khazaal, Y., Zullino, D., \& Clark, L. (2012). Trait gambling cognitions predict near-miss experiences and persistence in laboratory slot machine gambling. British Journal of Psychology, 103, 412-427. doi:10.1111/j.2044-8295.2011.02083.x

Bossuyt, E., Moors, A., \& De Houwer, J. (2014). Unexpected and just missed: The separate influence of the appraisals of expectancy and proximity on negative emotions. Emotion, 14, 284-300. doi:10.1037/a0034675

Chase, H. W., \& Clark, L. (2010). Gambling severity predicts midbrain response to near-miss outcomes. Journal of Neuroscience, 30, 6180-6187. doi:10.1523/JNEUROSCI.5758-09.2010

Chóliz, M. (2010). Experimental analysis of the game in pathological gamblers: Effect of the immediacy of the reward in slot machines. Journal of Gambling Studies, 26, 249-256.

Clark, L., Crooks, B., Clarke, R., Aitken, M. R. F., \& Dunn, B. D. (2012). Physiological responses to near-miss outcomes and personal control during simulated gambling. Journal of Gambling Studies, 28, 123-137. doi:10.1007/s10899-011-9247-z

Clark, L., Lawrence, A. J., Astley-Jones, F., \& Gray, N. (2009). Gambling near-misses enhance motivation to gamble and recruit win-related brain circuitry. Neuron, 61, 481-490. doi:10.1016/ j.neuron.2008.12.031

Côté, D., Caron, A., Aubert, J., Desrochers, V., \& Ladouceur, R. (2003). Near wins prolong gambling on a video lottery terminal. Journal of Gambling Studies, 19, 433-438.

Cyders, M. A., Smith, G. T., Spillane, N. S., Fischer, S., Annus, A. M., \& Peterson, C. (2007). Integration of impulsivity and positive mood to predict risky behavior: Development and validation of a measure of positive urgency. Psychological Assessment, 19, 107-118. doi:10. 1037/1040-3590.19.1.107

Dixon, M. J., Collins, K., Harrigan, K. A., Graydon, C., \& Fugelsang, J. A. (2015). Using sound to unmask losses disguised as wins in multiline slot machines. Journal of Gambling Studies, 31, $183-196$.

Dixon, M. J., Graydon, C., Harrigan, K. A., Wojtowicz, L., Siu, V., \& Fugelsang, J. (2014). The allure of multi-line games in modern slot machines. Addiction, 109, 1920-1928.

Dixon, M. J., Harrigan, K. A., Jarick, M., MacLaren, V., Fugelsang, J. A., \& Sheepy, E. (2011). Psychophysiological arousal signatures of near-misses in slot machine play. International Gambling Studies, 11, 393-407. doi:10.1080/14459795.2011.603134

Dixon, M. J., Harrigan, K. A., Sandhu, R., Collins, K., \& Fugelsang, J. A. (2010). Losses disguised as wins in modern multi-line video slot machines. Addiction, 105, 1819-1824. doi:10.1111/j.13600443.2010.03050.x

Dixon, M. J., Harrigan, K. A., Santesso, D. L., Graydon, C., Fugelsang, J. A., \& Collins, K. (2013a ). The impact of sound in modern multiline video slot machine play. Journal of Gambling Studies, 30, 913-929.

Dixon, M. J., MacLaren, V., Jarick, M., Fugelsang, J. A., \& Harrigan, K. A. (2013b). The frustrating effects of just missing the jackpot: Slot machine near-misses trigger large skin conductance responses, but no post-reinforcement pauses. Journal of Gambling Studies, 29, 661-674. doi:10. 1007/s10899-012-9333-x

Dixon, M. R., \& Schreiber, J. E. (2004). Near-miss effects on response latencies and win estimations of slot machine players. The Psychological Record, 54, 335-348.

Dow Schüll, N. (2012). Addiction by design: Machine gambling in Las Vegas. Princeton, NJ: Princeton University Press.

Dymond, S., Lawrence, N. S., Dunkley, B. T., Yuen, K. S., Hinton, E. C., Dixon, M. R., \& . . Singh, K. D. (2014). Almost winning: Induced MEG theta power in insula and orbitofrontal cortex increases during gambling near-misses and is associated with BOLD signal and gambling severity. NeuroImage, 91, 210-219. doi:10.1016/j.neuroimage.2014.01.019

Ferris, J., \& Wynne, H. (2001). The Canadian problem gambling index. Ottawa, ON: Canadian Centre on Substance Abuse.

Griffiths, M. (1993). Fruit machine gambling: The importance of structural characteristics. Journal of Gambling Studies, 9, 101-120. doi:10.1007/BF01014863

Harrigan, K., Dixon, M., MacLaren, V., Collins, K., \& Fugelsang, J. (2011). The maximum rewards at the minimum price: Reinforcement rates and payback percentages in multi-line slot machines. Journal of Gambling Issues, 26, 11-29. doi:10.4309/jgi.2011.26.3

Haw, J. (2008). Random-ratio schedules of reinforcement: The role of early wins and unreinforced trials. Journal of Gambling Issues, 21, 56-67. doi:10.4309/jgi.2008.21.6 
Jensen, C., Dixon, M. J., Harrigan, K. A., Sheepy, E., Fugelsang, J. A., \& Jarick, M. (2013). Misinterpreting 'winning' in multiline slot machine games. International Gambling Studies, 13, 112-126. doi:10.1080/14459795.2012.717635

Kassinove, J. I., \& Schare, M. L. (2001). Effects of the "near miss" and the "big win" on persistence at slot machine gambling. Psychology of Addictive Behaviors, 15, 155-158. doi:10.1037/0893164X.15.2.155

Ladouceur, R., Sévigny, S., Blaszczynski, A., O’Connor, K., \& Lavoie, M. E. (2003). Video lottery: Winning expectancies and arousal. Addiction, 98, 733-738. doi:10.1046/j.1360-0443.2003. 00412.x

Langer, E. J., \& Roth, J. (1975). Heads I win, tails it's chance: The illusion of control as a function of the sequence of outcomes in a purely chance task. Journal of Personality and Social Psychology, 32, 951-955. doi:10.1037/0022-3514.32.6.951

Loba, P., Stewart, S. H., Klein, R. M., \& Blackburn, J. R. (2001). Manipulations of the features of standard video lottery terminal (VLT) games: Effects in pathological and non-pathological gamblers. Journal of Gambling Studies, 17, 297-320. doi:10.1023/A:1013639729908

Markman, K. D., \& McMullen, M. N. (2003). A reflection and evaluation model of comparative thinking. Personality and Social Psychology Review, 7, 244-267. doi:10.1207/ S15327957PSPR0703_04

Meyer, G., Fiebig, M., Häfeli, J., \& Mörsen, C. (2011). Development of an assessment tool to evaluate the risk potential of different gambling types. International Gambling Studies, 11, 221-236. doi:10.1080/14459795.2011.584890

Michalczuk, R., Bowden-Jones, H., Verdejo-Garcia, A., \& Clark, L. (2011). Impulsivity and cognitive distortions in pathological gamblers attending the UK National Problem Gambling Clinic: A preliminary report. Psychological Medicine, 41, 2625-2635. doi:10.1017/ S003329171100095X

Parke, J., \& Griffiths, M. (2004). Gambling addiction and the evolution of the "near miss". Addiction Research and Theory, 12, 407-411. doi:10.1080/16066350410001728118

Qi, S., Ding, C., Song, Y., \& Yang, D. (2011). Neural correlates of near-misses effect in gambling. Neuroscience Letters, 493, 80-85. doi:10.1016/j.neulet.2011.01.059

Raylu, N., \& Oei, T. P. S. (2004). The Gambling Related Cognitions Scale (GRCS): Development, confirmatory factor validation and psychometric properties. Addiction, 99, 757-769. doi:10. 1111/j.1360-0443.2004.00753.x

Rockloff, M. J., \& Greer, N. (2011). Audience influence on EGM gambling: The protective effects of having others watch you play. Journal of Gambling Studies, 27, 443-451. doi:10.1007/s10899010-9213-1

Roese, N. J. (1997). Counterfactual thinking. Psychological Bulletin, 121, 133-148. doi:10.1037/ 0033-2909.121.1.133

Roese, N. J., \& Olson, J. M. (1995a). Counterfactual thinking: A critical overview. In N. J. Roese \& J. M. Olson (Eds.), What might have been: The social psychology of counterfactual thinking (pp. 1-59). Mahwah, NJ: Erlbaum.

Strickland, L. H., \& Grote, F. W. (1967). Temporal presentation of winning symbols and slotmachine playing. Journal of Experimental Psychology, 74, 10-13. doi:10.1037/h0024511

Thaler, R. H., \& Johnson, E. J. (1990). Gambling with the house money and trying to break even: The effects of prior outcomes on risky choice. Management Science, 36, 643-660. doi:10.1287/ mnsc.36.6.643

Weatherly, J. N., Sauter, J. M., \& King, B. M. (2004). The "big win" and resistance to extinction when gambling. The Journal of Psychology, 138, 495-504. doi:10.3200/JRLP.138.6.495-504 